\title{
Comparison of Karydakis Flap and Limberg Flap in Pilonidal
}

\section{Sinus}

\section{Ashok C* and Nikhilesh $\mathrm{K}$ \\ Department of Surgery, Tata Main Hospital, Jamshedpur, India}

*Corresponding author: Ashok Chattoraj MS, Head Consultant, Department of Surgery, Tata Main Hospital, Jamshedpur, India, Tel: +919431112883; Email: akchattoraj@gmail.com; drachattoraj@tatasteel.com

\section{Research Article}

Volume 2 Issue 2

Received Date: April 19, 2018

Published Date: May 26, 2018

\section{Abstract}

The occurrence of Pilonidal sinus in the community is quite common. This is a series of cases from Jan 2008 till Dec 2017. A total of 19 males were operated. They were in the age group 27 years to 45 years. All the individuals were hairy males. The patients were placed in 2 groups and treated by Limberg flap in one group and Karydakis flap in the second group. The surgical parameters taken included duration of surgery, duration of pain, length of hospital stay, return to work and post-operative result. 12 patients were treated by Limberg flap and 7 by Karydakis flap. The results in both groups were similar with the pain being lesser with Karydakis flap.

Keywords: Karydakis Flap; Surgery; Pilonidal sinus; Limberg flap

\section{Introduction}

Pilonidal sinus is a common and distressing condition affecting males more commonly than females. It is seen more commonly in hairy individuals and the natal cleft is the commonest site of involvement. Patients present with intermittent discharge of pus and serosanguinous fluid. It may be associated with abscess formation. The sinus tract commonly extends till the presacral fascia. There maybe 2 or more external openings. Excision of the sinus tract is the treatment in this condition. Several surgeries have been used to treat this condition. A recurrence rate of 7$22 \%$ is well documented.

\section{Materials and Methods}

A total of 19 patients were treated in the Department of Surgery, Tata Main Hospital, Jamshedpur from Jan 2008 till Dec 2017. The patients were explained about the procedures and divided into 2 groups-

a) First group of 12 patients were treated by Limberg flap (Figures 1-3).

b) Second group of 7 patients were treated by Karydakis flap (Figures 4 \& 5).

All the 19 patients were males ranging from $27-45$ years age (Table 1 ). The median age was 34 years. 8 patients were well built, 5 patients were obese and 6 patients were thin built. The outcome parameters were taken as follows- duration of surgery, duration of pain, length of hospital stay, return to work and post-operative result. 


\section{International Journal of Surgery \& Surgical Techniques}

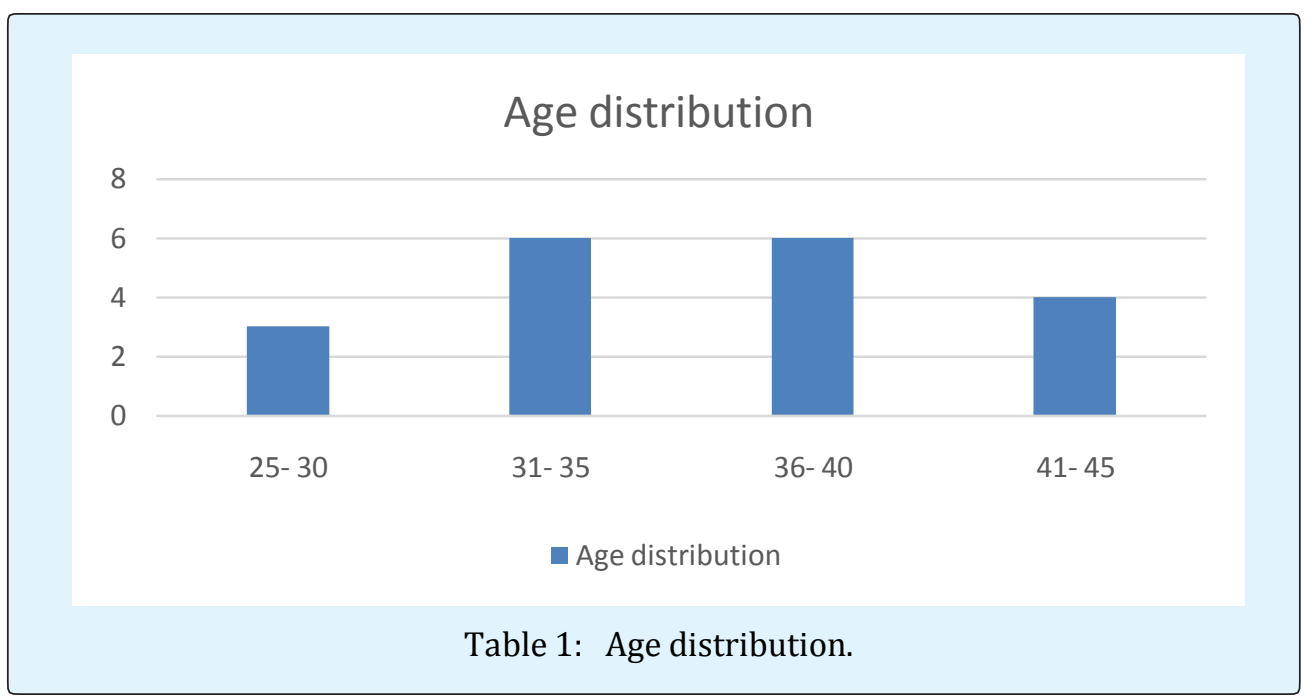

These patients were admitted one day prior to surgery. Part shaving was done on the morning of the surgery. Preoperative antibiotic of Inj. Ceftriaxone- $1 \mathrm{Gm}$ IV was used for 3 doses; first at the time of induction and 2 doses postoperatively. 15 patients were administered Spinal anaesthesia and 4 patients were given general anaesthesia because of patient choice. The position of the patient on table was prone position with bolsters below the chest and pelvis areas.

\section{Limberg flap}

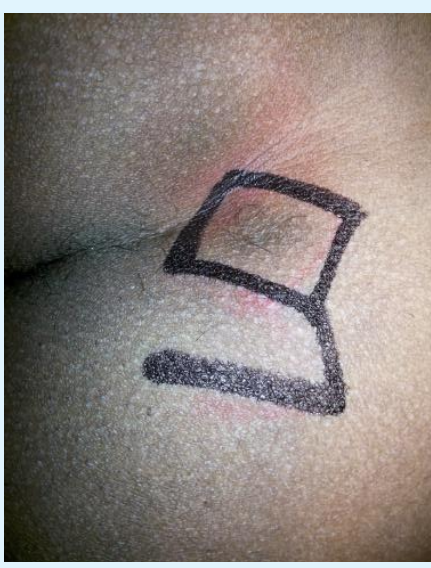

Figure 1

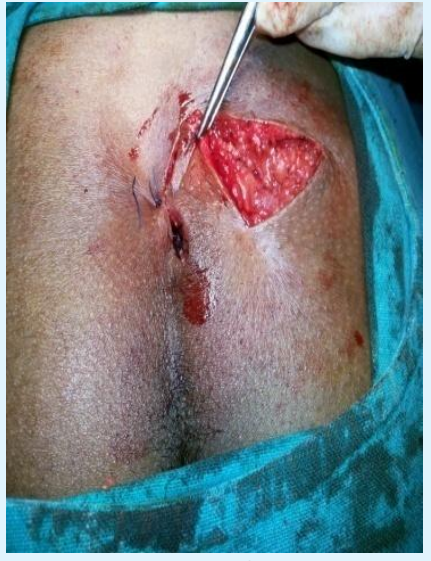

Figure 2

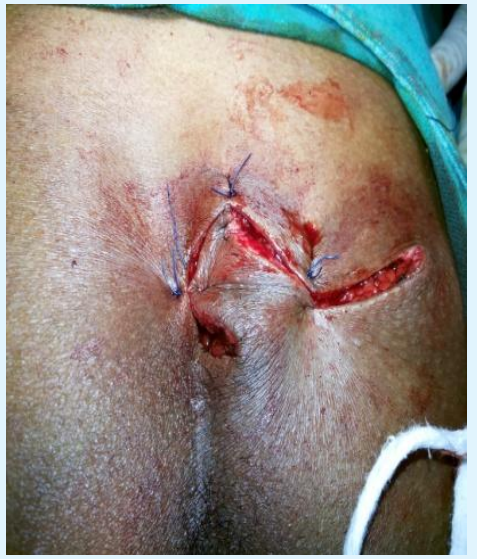

Figure 3

Figure 1:Operative marking of Limberg flap

Figure 2: Limberg flap dissection in progress

Figure 3: Completed Limberg flap 


\section{International Journal of Surgery \& Surgical Techniques}

\section{Karydakis flap}

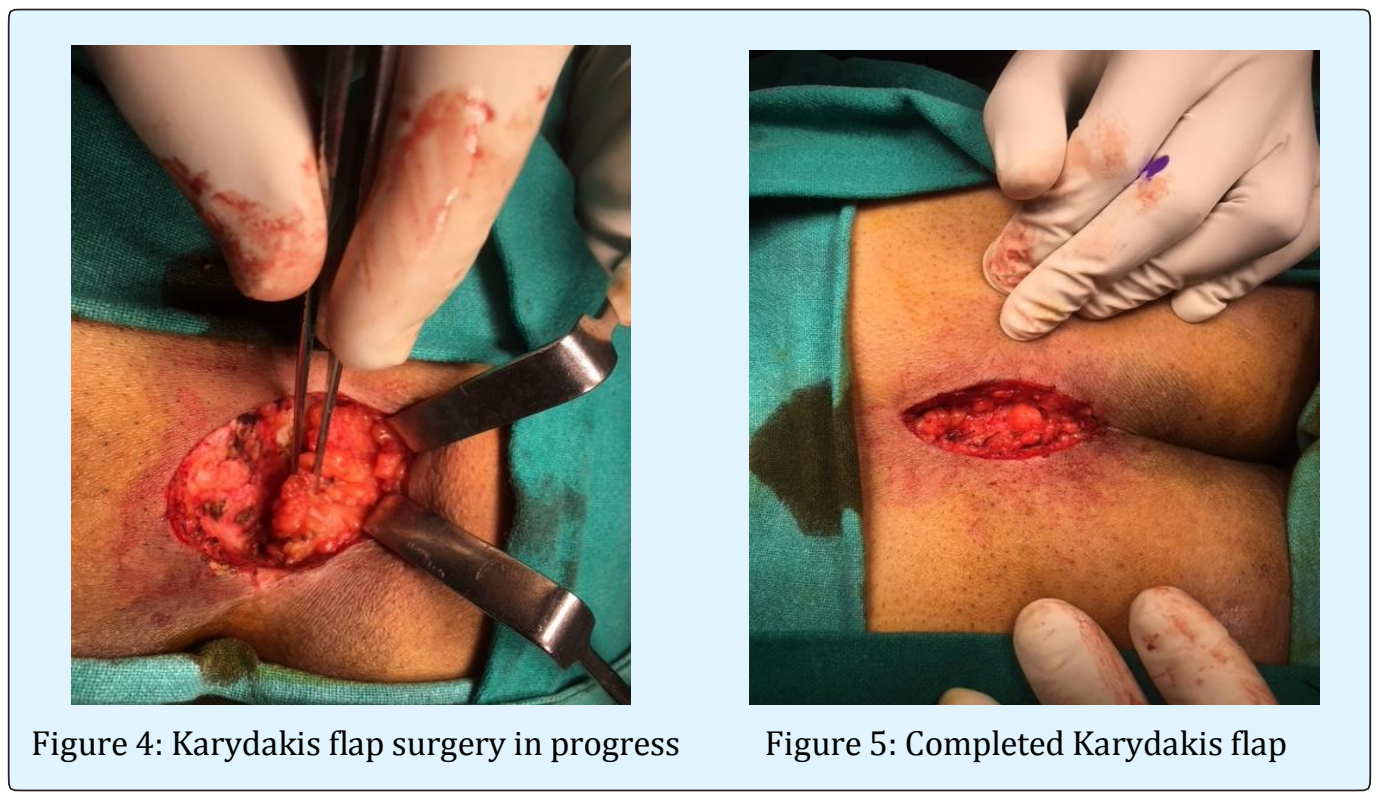

\section{Observation}

The time duration for Limberg flap was $1 \mathrm{hr} 15 \mathrm{mins} \pm$ 15 mins and for Karydakis flap was 50 mins \pm 15 mins. The operative blood loss was $<50 \mathrm{ml}$ in both groups. Patients with Limberg flap had pain as per VAS score for 48 hours whereas in the Karydakis group it was 40 hours. All the patients were discharged on the $3^{\text {rd }}$ post-operative day after the wound was inspected. The wound was again inspected on the $7^{\text {th }}$ post-operative day and the skin sutures were removed on the $19^{\text {th }}$ day.

\section{Results}

2 patients had mild redness over the scar site. There was no discharge or collection in any of these patients.

12 patients returned to work on the $17^{\text {th }}$ post-operative day, 4 on the $20^{\text {th }}$ day and the remaining 3 joined work after 1 month of the surgery.

The patients had no complaints on their follow up visit 2 months after surgery.

The histopathology of all the sinus tracts were chronic inflammation. No evidence of TB.

\section{Discussion}

Pilonidal sinus is a common condition usually found with young population especially in men which occurs in the 3rd decade and above. It often occurs in the sacrococcygeal region. Its etiology has not been clearly explained yet. There are some predisposing factors for its occurrence. Some of these factors are hairy sacrococcygeal area, sitting on the firm ground for a long time, narrow and deep natal cleft and humid skin [1].

There are two aims in management of pilonidal sinus disease. First, excision with effective healing and minimal recurrence rate. Second aim is low post surgical morbidity in minimizing hospitalization period and loss of workdays causing minimal inconvenience to the patient. The adopted method for treatment should aim at these goals [2,3]. Although several surgical methods have been defined for PSD treatment, no golden standard method is available yet.

Marsupialisation was being used in surgical pilonidal sinus treatment for long. Marsupializing the edges of the wound used to be done to ensure reduction in wound size and shorten healing time. Nevertheless, it is noteworthy to remember that healing time of the patients who received marsupialization lasts up to 4 to 5 weeks, and a significant number of wound dressings was required as a result [4]. The disadvantages mentioned led the surgeons to abandon the practice of leaving the wound open, and practice operations such as excision and primary repair. These operations are destined to ensure shorter returnto-work time and quick-healing compared with marsupialization, yet it is reported that they have high complication rates (wound disintegration, infection, pain) $[5,6]$. 


\section{International Journal of Surgery \& Surgical Techniques}

The present study compares two flap procedures; modified Limberg flap and Karydakis flap in the surgical management of sacrococcygeal pilonidal sinus disease. We found no significant differences between the groups in short term results regarding operation time, patient satisfaction of cosmetic results and time to return to normal activities.

Bostanoglu et al. demonstrated that hospital stay was shorter (3.0 \pm 1.5 days) in Karydakis technique as compared to Limberg flap procedure (4.3 \pm 1.5 days) [7] The study carried out by Can, et al. on 145 patients did not discover significant differences between the two groups (MLF group, $n=72$; KF group, $n=73$ ) in terms of wound infection, collection, wound disintegration, and total surgical area complications considering early postop findings [8]. Karaca, et al. carried out a prospective randomized study on 81 patients (MLF, $n=46$; MKF, $n=$ 35) where they found that MLF operations are superior to MKF operations in terms of postoperative pain, time of painless sitting on chair and time of painless sitting on armchair. The same study further found no significant difference between the groups in terms of full healing time [9].

\section{References}

1. Doll D, Friederichs J, Dettmann H, Boulesteix AL, Duesel W, et al. (2008) Time and rate of sinus formation in pilonidal sinus disease. Int J Colorectal Dis 23(4): 359-364.

2. Spivak H, Brooks V, Nussbaum M (1996) Treatment of chronic pilonidal sinus. Dis Colon Rectum 39: 11361139.
3. Katsoulis IE, Hibberts F, Carapeti EA (2006) Outcome of treatment of primary and recurrent pilonidal sinuses with the Limberg flap. Surgeon 4(1): 7-10.

4. Mentes O, Bagci M, Bilgin T, Coskun I, Ozgul O, et al. (2005) Management of pilonidal sinus disease with oblique excision and primary closure: Result of 493 patients. Dis Colon Rectum 49(1): 104-108.

5. Schoeller T, Wechselberger G, Otto A, Papp C (1997) Definite surgical treatment of complicated recurrent pilonidal disease with a modified fasciocutaneous $\mathrm{V}-\mathrm{Y}$ advancement flap. Surgery 121(3): 258-263.

6. Ertan T, Koc M, Gocmen E, Aslar K, Keksek M, et al. (2005) Does technique alters quality of life after pilonidal sinus surgery? Am J Surg 190(3): 388-392.

7. Bostanoglu S, Sakcak I, Avsar FM, Cosgun E, Hamamci EO (2010) Comparison of Karydakis technique with Limberg Flap Procedure in Pilonidal Sinus Disease: Advantages of Karydakis technique. Pak J Med Sci 26(4): 773-777.

8. Can MF, Sevinc MM, Hancerliogullari O, Yilmaz M, Yagci G (2010) Multicenter prospective randomized trial comparing modified Limberg flap transposition and Karydakis flap reconstruction in patients with sacrococcygeal pilonidal disease. Am J Surg 200(3): 318-327.

9. Karaca T, Yoldaş O, Bilgin BÇ, Ozer S, Yoldaş S, et al. (2012) Comparison of short-term results of modified Karydakis flap and modified Limberg flap for pilonidal sinus surgery. Int J Surg 10(10): 601-606.

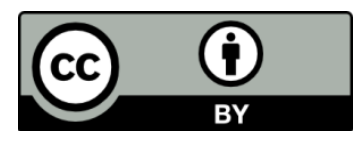

